\title{
Gancao (Glycyrrhizae Radix) provides the main contribution to Shaoyao-Gancao decoction on enhancements of CYP3A4 and MDR1 expression via pregnane $\mathrm{X}$ receptor pathway in vitro
}

\author{
Dandan Feng ${ }^{1}$, Tao Tang ${ }^{1}$, Rong Fan ${ }^{1}$, Jiekun Luo ${ }^{1}$, Hanjin Cui ${ }^{1}$, Yang Wang ${ }^{1 *}$ (D) and Pingping Gan²*
}

\begin{abstract}
Background: Chinese herbal formula Shaoyao Gancao decoction (SGD) is often used as an adjuvant with chemotherapeutic agents to treat cancer. Due to the herb-drug interactions, the alternations of drug metabolic enzyme and drug transporters induced by SGD deserve to be explored. We aimed to investigate the effect of SGD on the pregnane $X$ receptor (PXR)-mediated transcriptional regulation of cytochrome P450 3A4 (CYP3A4) and drug transporter multidrug resistance protein 1 (MDR1) in vitro. Besides, we assessed the contribution of constituent herbs to SGD on the regulation of CYP3A4 and MDR1.

Methods: The dual luciferase reporter gene system containing the hPXR expression plasmid and the reporter gene plasmid of CYP3A4 or MDR1 was co-transfected to HepG2 and Caco2 cells. Luciferase activities were determined using a Dual-luciferase reporter assay kit. The gene expression of CYP3A4 and MDR1 in the hPXRtransfected LS174T cells were assessed by real-time qPCR. Finally, the contribution of constituent herbs from SGD was evaluated.

Results: SGD, Shaoyao and Gancao concentration-dependently increased promoter activities of CYP3A4 and MDR1 in vitro. Moreover, SGD, Shaoyao and Gancao up-regulated CYP3A4 and MDR1 mRNA in hPXR-transfected LS174T cells. As the herbal constituent of SGD, Gancao possesses significantly higher levels of metabolic enzyme and drug transporters compared with Shaoyao.

Conclusion: SGD tends to enhance CYP3A4 and MDR1 expression via PXR pathway, especially Gancao provides the main contribution. This study highlights a potential in vitro mechanism for SGD on the regulation of drug metabolic enzymes and drug transporters.
\end{abstract}

Keywords: Shaoyao Gancao decoction, Pregnane X receptor, Cytochrome P450 3A4, Multidrug resistance protein 1, Traditional Chinese medicine

\footnotetext{
* Correspondence: wangyang_xy87@csu.edu.cn; gppxy@csu.edu.cn

${ }^{1}$ Institute of Integrative Medicine, Xiangya Hospital, Central South University, Changsha 410008, People's Republic of China

2Department of Oncology, Xiangya Hospital, Central South University,

Changsha 410008, People's Republic of China
}

(c) The Author(s). 2018 Open Access This article is distributed under the terms of the Creative Commons Attribution 4.0 International License (http://creativecommons.org/licenses/by/4.0/), which permits unrestricted use, distribution, and reproduction in any medium, provided you give appropriate credit to the original author(s) and the source, provide a link to the Creative Commons license, and indicate if changes were made. The Creative Commons Public Domain Dedication waiver (http://creativecommons.org/publicdomain/zero/1.0/) applies to the data made available in this article, unless otherwise stated. 


\section{Background}

Chinese herbal medicine becomes the main adjuvant combined with chemotherapy to improve cancer patients' quality of life $[1,2]$. An overall prevalence of herbal formulas use is estimated from 13 to $63 \%$ among cancer patients in the United States [3]. Herbal medicine can enhance the response rate or chemosensitivity, reduce side-effects of chemotherapy, and prolong the survival time of cancer patients [4-6]. To date, a number of herbs and herbal formulas as an adjuvant have been reported to be beneficial to cancer patients, such as PHY906 (Huangqin Tang) [7], Liu Jun Zi Tang (Rikkunshito in Japanese) [8], and Shaoyao Gancao decoction (shakuyaku-kanzo-to in Japanese) [9].

Shaoyao Gancao decoction (SGD), a classical analgesic prescription, is composed of Shaoyao (Paeoniae Radix Alba) and Gancao (Glycyrrhizae Radix et Rhizoma) in the ratio of 1:1. It is originated from the Treatise on Febrile Diseases, and is widely used in Asia to relieve menstrual pain, muscle spasm, and muscle pain [10, 11]. In Japan and China, doctors prefer to prescribe SGD with chemotherapeutic agent paclitaxel to relieve paclitaxel-induced myalgia and arthralgia [9, 12], and painful peripheral neuropathy $[13,14]$. However, in view of the narrow therapeutic window of chemotherapeutic agents, this concomitant use increases the risk of clinically relevant herb-antineoplastic agent interactions [15]. While reducing the toxic side effects of paclitaxel, SGD also increases the metabolism of paclitaxel [15]. Our previous study has proved that pretreatment with SGD for 14 consecutive days significantly decreases the area under the curve and increases the total clearance of intravenous paclitaxel in rats [15]. Most known herb-drug interactions are due to changes in metabolic routes, which is related to altered expression or functionality of drug metabolic enzymes and/or drug transporters $[3,16,17]$. Therefore, it is quite necessary to evaluate effects of SGD on drug metabolic enzymes and drug transporters.

Cytochrome P450 (CYP450) occupies an important role in the metabolism and detoxification process of drug and other endo-or xeno-biotics. The largest fraction of the CYP450 CYP3A4, representing 40\% of the total hepatic and $80 \%$ of the total intestinal CYPs $[18,19]$, is involved in the metabolism of approximately $50 \%$ of marketed drugs [20]. Drug transporter multidrug resistance protein 1 (MDR1) encodes P-glycoprotein, an efflux pump, and then plays an important role in the absorption and presystemic elimination of many xenobiotics [21]. Furthermore, the regulation of CYP3A4 and MDR1 is mediated primarily through the activation of nuclear xenobiotic pregnane $\mathrm{X}$ receptor (PXR) $[22,23]$. The ethanol extracts of Shaoyao or Gancao have been shown to activate human PXR and induce CYP3A4 reporter constructs in HepG2 cells [24]. The aqueous extracts of Shaoyao or Gancao have been reported to induce
P-glycoprotein-mediated drug transport [23, 25, 26]. Therefore, we speculate that the mechanism underlying the interaction from SGD might be related to regulation of CYP3A4 and/or MDR1 expression via PXR pathway.

In this study, human hepatoma cell line HepG2 and colon carcinoma cell lines $\mathrm{Caco} 2$ were used to determine the potential of SGD to induce PXR-mediated transcriptional regulation of CYP3A4 and MDR1 in vitro. Cells were co-transfected with a PXR-expressed CYP3A4 or MDR1 dual luciferase reporter gene constructs and then exposed to a range of SGD, or Shaoyao (SY), or Gancao (GC). Luciferase activities were determined using a Dual-luciferase reporter assay kit. In addition, the effect of SGD on CYP3A4 and MDR1 mRNA expression in PXR-transfected LS174T cells was measured using real-time qPCR. The present study also investigated the contribution of constituent herbs Shaoyao and Gancao to SGD. The workflow is illustrated in Fig. 1.

\section{Methods \\ Chemicals}

Rifampicin (RIF), pregnenolone 16 $\alpha$-carbonitrile(PCN), phenobarbital (PB), dimethyl sulfoxide (DMSO), diethyl pyrocarbonate (DEPC) were purchased from Sigma-Aldrich (St. Louis, MO, USA). RIF, PCN, and PB were dissolved in DMSO at concentrations appropriate for the specific studies in which they were used. Trypsin and Taq DNA polymerase were purchased from Gibco BRL (Grand Island, NY, USA). Reverse transcription system, dual-luciferase reporter assay system, pGL4.17-Luc and pGEM-T constructs were purchased from Promega (Madison, WI, USA).

\section{Preparation of SGD, SY and GC}

Shaoyao (Paeoniae Radix Alba, the root of Paeonia lactiflora Pall.) and Gancao (Glycyrrhizae Radix et Rhizoma, the root and rhizome of Glycyrrhiza uralensis Fisch.) were purchased from Xiangya Hospital of Central south university (Changsha, China). The crude herbs were authenticated by Prof. SY Hu, Department of Chinese Herbal Medicine of Central South University. Voucher specimens (No. 20140505 and No. 20140506) were deposited at the Laboratory of Ethnopharmacology in Xiangya Hospital. Crude drugs of SGD (SY: GC, 1:1, w/ w) $200 \mathrm{~g}$ were immersed in $400 \mathrm{ml}$ distilled water for 30 min and boiled for $40 \mathrm{~min}$, then filtered. The residue was boiled and filtered once more. The filtrates from each time were combined and centrifuged at $3000 \mathrm{rpm}$ for $30 \mathrm{~min}$ at room temperature. The supernatant was filtered through a $0.22-\mu \mathrm{m}$ filter. The concentration of the original SGD was adjusted to $500 \mathrm{mg}$ crude drug per milliliter, and then diluted into $0.1,1,2 \mathrm{mg} / \mathrm{ml}$. SY (Paeoniae Radix Alba 100 g) or GC (Glycyrrhizae Radix et Rhizoma $100 \mathrm{~g}$ ) was immersed in $400 \mathrm{ml}$ distilled 


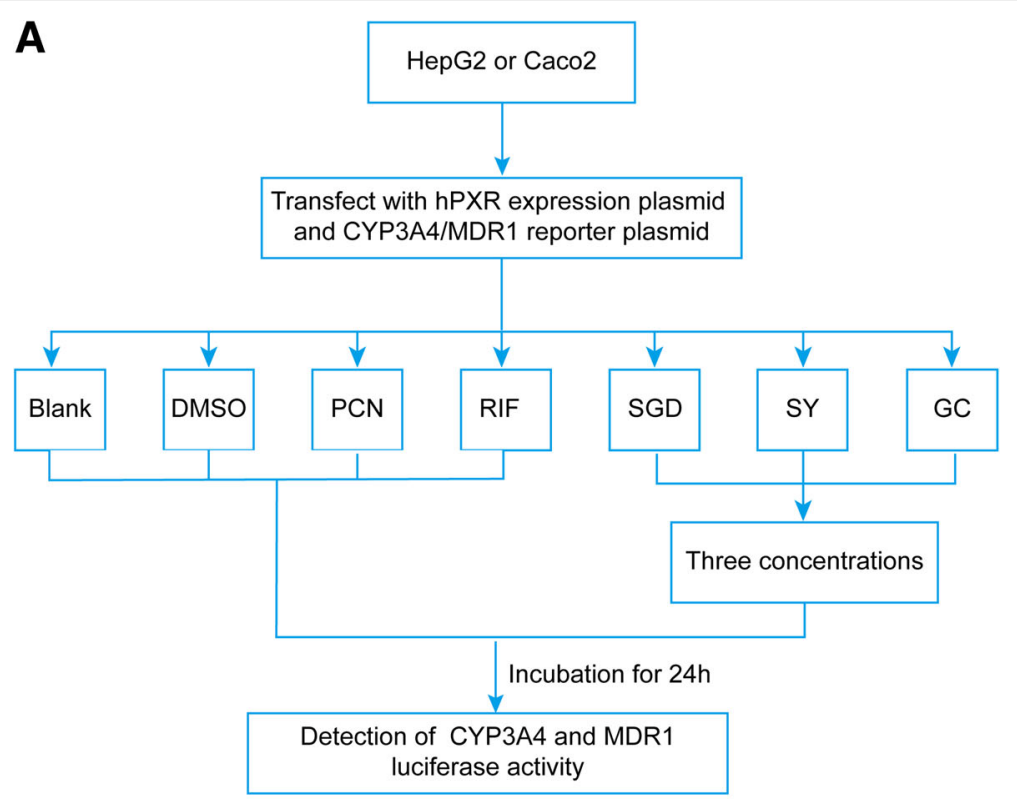

B

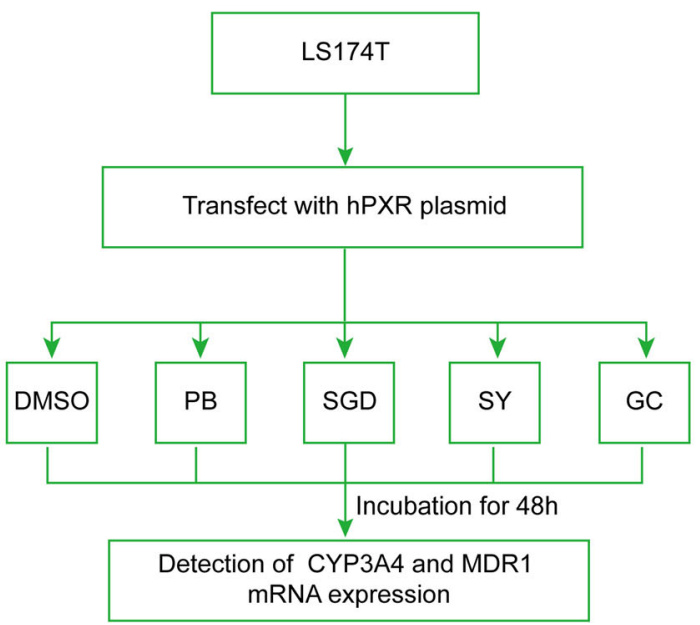

Fig. 1 The workflow of the present study. a The workflow of the study that whether SGD regulates CYP3A4 or MDR1 luciferase activities through hPXR. HepG2 and Caco2 cells were co-transfected with a PXR-expressed CYP3A4 or MDR1 dual luciferase reporter gene constructs and then exposed to a range of SGD, or Shaoyao (SY), or Gancao (GC). After $24 \mathrm{~h}$, luciferase activities were evaluated. b The workflow of the study that whether SGD regulates CYP3A4 or MDR1 mRNA expression through hPXR. LS174T cells were co-transfected with PXR plasmid and then exposed to SGD, or Shaoyao (SY), or Gancao (GC). After 48 h, CYP3A4 and MDR1 mRNA expression were measured

water, and processed as SGD. The concentration of the original SY and GC was adjusted to $250 \mathrm{mg}$ crude drug per milliliter, and then diluted into $0.05,0.5,1 \mathrm{mg} / \mathrm{ml}$. Decoctions were maintained at $4{ }^{\circ} \mathrm{C}$ for further use.

Meanwhile, six key compounds (gallic acid, albiflorin, paeoniflorin, glycyrrhizin, benzoic acid and liquiritigenin) from SGD for quality control using a Waters Acquity ultra-performance liquid chromatography (UPLC) system have been reported in our previous studies [27-29]. Among the six compounds, gallic acid, albiflorin, paeoniflorin and benzoic acid are for SY, while glycyrrhizin and liquiritigenin are for GC.

\section{Cell culture}

Three cell lines were used in this study including HepG2 (human Caucasian hepatocellular carcinoma), Caco2 (human colon adenocarcinoma) and LS174T (human colon adenocarcinoma). Three cell lines were purchased from the Shanghai Institute of Cell Biology, Chinese Academy of Sciences (Shanghai, China). The HepG2 line was maintained in Dulbecco's modified Eagle medium (DMEM) high glucose medium (HyClone), Caco2 and LS174T lines were maintained in DMEM/F12 medium (HyClone), at $37^{\circ} \mathrm{C}$ in a humidified atmosphere containing $5 \% \mathrm{CO}_{2}$. All media were supplemented with $10 \%$ 
fetal bovine serum (FBS, Gibco), added with $100 \mathrm{U} / \mathrm{ml}$ penicillin and $100 \mathrm{U} / \mathrm{ml}$ streptomycin (Beyotime, Shanghai, China). The medium was replaced every 3 days.

\section{Plasmids construction}

The expression plasmid for the human PXR receptor pcDNA3.1-PXR was kindly provided by Prof. Guo Wang (Institute of Clinical Pharmacology, Central South University, Changsha, China), containing the full-length human PXR [30].

The CYP3A4-Luc reporter constructs containing the basal promoter $(-362 \sim+53 \mathrm{bp})$ with proximal PXR response element and the distal xenobiotic responsive enhancer module $(-7836 \sim-7208 \mathrm{bp})$ of the CYP3A4 gene 5 '-flanking region inserted to pGL4.17-Basic reporter vector has been reported previously [30]. The construction of MDR1-Luc reporter gene vector has been described previously $[21,30]$. Human MDR1 promoter fragment $(-7975 \sim-7013 \mathrm{bp})$ containing the cluster of xenobiotic-responsive enhancer modules was amplified by PCR with primers 5'- TCTGCTAGC AGTGTTTCTTGT-3', containing a natural NheI site, and $5^{\prime}$-AATCTCGAGCATATAAGGCAACTGTTTTG T-3', introducing an XhoI site. The NheI/XhoI-digested PCR fragment was ligated between the NheI/XhoI sites of pGL4.17-Basic luciferase reporter vector.

\section{Cell transient transfections}

Transient transfections of HepG2 and Caco2 cells were performed as described previously [30]. In brief, prior to cell transfection for $24 \mathrm{~h}, \mathrm{HepG} 2$ or Caco2 cells were seeded in 24-well and 6-well plates at a density of $1 \times 10^{5}$ cells per well. Transfections were performed in triplicate using $600 \mathrm{ng}$ CYP3A4/MDR1-Luc, $100 \mathrm{ng}$ pcDNA3.1-PXR (or $100 \mathrm{ng}$ pcDNA3.1), $10 \mathrm{ng}$ internal control PRL-SV40, and $5 \mu \mathrm{l}$ lipofectamine 2000 (Invitrogen) in a well. Four to six hours after transfection, HepG2 cells were washed in DMEM, replaced with DMEM + 10\% FBS medium and treated with drugs $24 \mathrm{~h}$ later, while Caco2 cells were washed in DMEM/F12, replaced with DMEM/F12 + 10\% FBS medium and treated with drugs $24 \mathrm{~h}$ later.

LS174T cells $\left(1.2 \times 10^{5}\right.$ per well) were seeded into 24-well and 6-well plates and cultivated for $24 \mathrm{~h}$. Then LS174T cells were transfected with $100 \mathrm{ng} /$ well PXR expression plasmids or pcDNA3.1 vector or weren't transfected. Four to six hours after transfection, LS174T cells were washed in DMEM/F12, replaced with DMEM/F12 + 10\% FBS medium and treated with drugs 24h later.

\section{Western blot analysis}

After transfection, we firstly detected expression of PXR protein in non-transfected, vector-transfected, and PXR-transfected cells by western blot. As for HepG2 or
Caco2 cells in 6-well plates, we set non-transfected cells as the blank group, cells transfected with $600 \mathrm{ng}$ pGL4.17-CYP3A4 (or MDR1) + 100 ng pcDNA3.1 + 10 ng PRL-SV40 as the vector group, cells transfected with 600 ng pGL4.17-CYP3A4 (or MDR1) + 100 ng pcDNA3.1-PXR +10 ng PRL-SV40 as the trans-PXR group. As for LS174T cells in 6-well plates, we set non-transfected cells as the blank group, cells transfected with $100 \mathrm{ng}$ pcDNA3.1 + 10 ng PRL-SV40 as the vector group, cells transfected with $100 \mathrm{ng}$ pcDNA3.1-PXR + $10 \mathrm{ng}$ PRL-SV40 as the trans-PXR group.

Western blot analysis was performed according to the standard protocol. Following $48 \mathrm{~h}$ transfection, transfected cells were washed with cold phosphate buffer saline once and lysed using $50 \mu \mathrm{l}$ radioimmunoprecipitation assay lysis buffer (Pierce; Thermo Fisher Scientific, Inc.) according to the manufacturer's protocol. After $30 \mathrm{~min}$ on ice, lysates were clarified by centrifugation at $12000 \times \mathrm{rpm}$ for $15 \mathrm{~min}$ at $4{ }^{\circ} \mathrm{C}$. Amounts of protein $50-100 \mu \mathrm{g}$ were loaded on a $10 \%$ SDS-PAGE and subsequently transferred to polyvinylidene difluoride membranes. Following a blocking incubation with TBST containing 5\% non-fat dried milk at room temperature for $2 \mathrm{~h}$, membranes were incubated at $4{ }^{\circ} \mathrm{C}$ overnight with primary antibody (1:1000 dilution) to PXR (rabbit, no. 15607-1-AP, Proteintech) or $\beta$-actin (mouse, no. 60008-1-Ig, Proteintech), followed by incubation at room temperature for $1 \mathrm{~h}$ with the goat anti-mouse or anti-rabbit horseradish peroxidase conjugated secondary antibody (1,3000 dilution; cat no. ab97051, Proteintech). The membranes were analyzed using ECL detection reagent (Thermo). $\beta$-actin was used as an internal control. All experiments were conducted in triplicate.

\section{Herbal treatment in HepG2 or Caco2 cells and detecting luciferase activity}

Co-transfected HepG2 or Caco2 cells were treated with rifampicin $(10 \mu \mathrm{M}), \mathrm{PCN}(10 \mu \mathrm{M}), \operatorname{SGD}(0.1,1$, and 2 $\mathrm{mg} / \mathrm{ml})$, SY $(0.05,0.5$, and $1 \mathrm{mg} / \mathrm{ml}), \mathrm{GC}(0.05,0.5$, and $1 \mathrm{mg} / \mathrm{ml})$, or the solvent control (0.1\% DMSO) for $24 \mathrm{~h}$, respectively. Vector control (VC) group was transfected with pcDNA3.1-PXR + PRL-SV40 + pGL4.17 (or pcDN A3.1 + PRL-SV40 + pGL4.17-CYP3A4) and treated with $0.1 \%$ DMSO. The blank group was co-transfected with pcDNA3.1-PXR + PRL-SV40 + pGL4.17-CYP3A4 and treated with nothing. The firefly luciferase activity was detected using the dual-luciferase reporter assay (Promega) according to the manufacturer's protocol. The Renilla luciferase activity was used as an internal control. Each experiment was repeated three times in triplicate. The efficiency of transfection in each treatment groups was adjusted by the ratio of firefly luciferase activity to Renilla luciferase activity. 


\section{Real-time qPCR analysis of CYP3A4 and MDR1 mRNA in LS174T}

To determine CYP3A4 and MDR1 mRNA induction, LS174T cells were transfected with $100 \mathrm{ng} /$ well PXR expression plasmids or pcDNA3.1 vector or weren't transfected. Following $24 \mathrm{~h}$ transfection, appropriate cell samples were exposed to phenobarbital $(1 \mathrm{mM})$, SGD $(1 \mathrm{mg} / \mathrm{ml}), S Y(1 \mathrm{mg} / \mathrm{ml}), \mathrm{GC}(1 \mathrm{mg} / \mathrm{ml})$ or the solvent control (0.1\% DMSO) for $48 \mathrm{~h}$, respectively. Then cells were harvested and the mRNA levels of CYP3A4, MDR1 were measured by real-time qPCR.

Total RNA isolation was performed using TRIzol reagent (Invitrogen) and converted into cDNA using the Fermentas RT kit according to the manufacturer's instructions. The primers designed using Primer Premier 5.0 are presented in Table 1. PCR was performed in a total reaction volume of $30 \mu \mathrm{L}$, containing $15 \mu \mathrm{L} 2 \times$ SYBR Green PCR Master Mix (Applied Biosystems), $3 \mu \mathrm{L}$ cDNA, $1 \mu \mathrm{L}$ forward primer $(10 \mu \mathrm{M}), \quad 1 \mu \mathrm{L}$ reverse primer $(10 \mu \mathrm{M})$, and $10 \mu \mathrm{L}$ double-distilled water. Real-Time qPCR analysis was performed using the Applied Biosystems 7500 real-time PCR system. The temperature profile was $95^{\circ} \mathrm{C}$ for $10 \mathrm{~min} ; 40 \mathrm{cy}-$ cles of $95^{\circ} \mathrm{C}$ for $10 \mathrm{~s}, 59^{\circ} \mathrm{C}$ for $50 \mathrm{~s}$; melting curve program $60-95^{\circ} \mathrm{C}$. Real-time qPCR for each gene of interest was performed in triplicate, and the expression values were calculated as their average. Gene expression was evaluated in at least three independent experiments and each performed in triplicates. The expression of the target genes were normalized to the reference gene $\beta$-actin and then processed using the $2^{-\Delta \Delta \mathrm{Ct}}$ method [31]. The data are expressed as the fold changes in the activation of gene expression relative to the non-transfected group with $0.1 \%$ DMSO treatment (set to be 1).

\section{Statistical analysis}

All data are expressed as means \pm S.E.M of three independent experiments performed in triplicates. The data were first tested for the normality of distribution with Shapiro-Wilk test. The distribution of samples is approximately normal. Differences between groups were analyzed using a one-way ANOVA with Welch correction, followed by the Games Howell test. All statistical analyses were performed using SPSS 23.0 (International

Table 1 Primer sequences used for real-time qPCR

\begin{tabular}{lll}
\hline Gene & Orientation & 5'-3' sequence \\
\hline CYP3A4 & Forward & 5'-GCACCGAGTGGATTCCTT-3' \\
& Reverse & 5'-GGACATCAGGGTGAGTGGC-3' \\
MDR1 & Forward & 5'-AGGCTCGCCAATGATGC-3' \\
& Reverse & 5'-TCCTGTCCCAAGATTTGCTAT-3' \\
$\beta$-actin & Forward & 5'-CATCCTGCGTCTGGACCTGG-3' \\
& Reverse & 5'-TAATGTCACGCACGATTTCC-3' \\
\hline
\end{tabular}

Business Machines Corp., Armonk, NY, USA). Values of $p<0.05$ are considered to be statistically significant.

\section{Results}

SGD and its constituent herbs significantly enhance the CYP3A4 promoter activities via hPXR in HepG2 and Caco2 cells

First, we examined whether SGD and its constituent prescriptions (SY, GC) affect the activation of CYP3A4-Luc reporter constructs through hPXR. Since CYP3A4 is mainly distributed in the liver and intestine organs, we used the two human tumor cell lines [32]. We successfully transfected PXR-CYP3A4 plasmid constructs to HepG2 and Caco2 cells (Fig. 2). Twenty-four hours following co-transfection, HepG2 and Caco2 cells were separately treated with rifampicin $(10 \mu \mathrm{M}, \mathrm{hPXR}$ agonist), PCN (10 $\mu \mathrm{M}$, rodent PXR agonist), 0.1, 1, $2 \mathrm{mg} / \mathrm{ml} \mathrm{SGD} \mathrm{or} 0.05$, $0.5,1 \mathrm{mg} / \mathrm{ml}$ constituent prescriptions (SY and GC) for 24 $\mathrm{h}$, then detected dual luciferase activity.

Results of specific activity values of various concentrations groups are presented in Fig. 3. In HepG2 and Caco2 cells co-transfected with hPXR expression plasmid, rifampicin, SGD and its constituent prescriptions significantly increase the luciferase activities of CYP3A4 when compared with $0.1 \%$ DMSO vehicle control group $(p<0.05)$. In HepG2 cells transfected with PXR expression plasmid, rifampicin $(10 \mu \mathrm{M})$, SGD $(0.1$ and $2 \mathrm{mg}$ / $\mathrm{ml})$, SY $(0.05,0.5$, and $1 \mathrm{mg} / \mathrm{ml})$ and $\mathrm{GC}(0.05,0.5$, and $1 \mathrm{mg} / \mathrm{ml})$ markedly increase CYP3A4 luciferase activity by $24.80,(8.27,12.05),(5.24,5.74,7.12),(12.68,13.86$, 16.85)-fold compared with DMSO, respectively. While 1 $\mathrm{mg} / \mathrm{ml}$ SGD increase 10.00-fold CYP3A4 luciferase activity to the DMSO group without a statistical significance. In Caco2 cells transfected with PXR expression plasmid, CYP3A4 luciferase activity is significantly increased by rifampicin $(22.26)$, SGD $(8.62,10.75,13.40)$, $1 \mathrm{mg} / \mathrm{ml} \mathrm{SY}$ (9.24), GC (13.87, 14.53, 17.83) when compared with DMSO, respectively. The increases in CYP3A4 luciferase activities by three decoctions show a concentration-dependent trend.

However, in both transfected HepG2 and Caco2 cells, PCN treatment doesn't cause a significant increase in CYP3A4 luciferase activities when compared with the DMSO group. Previous studies have found rifampicin activates human but not rodent PXR, while PCN activates rodent but not human PXR [33, 34]. Consistent with the previous report [35], similar activation of hPXR and inductions of CYP3A4 and MDR1 by rifampicin are observed in HepG2, Caco-2 cells, except PCN.

SGD and its constituent herb GC markedly elevate the MDR1 promoter activities via hPXR in HepG2 and Caco2 cells Then, we examined whether the three decoctions induce the activation of MDR1-Luc reporter constructs through 


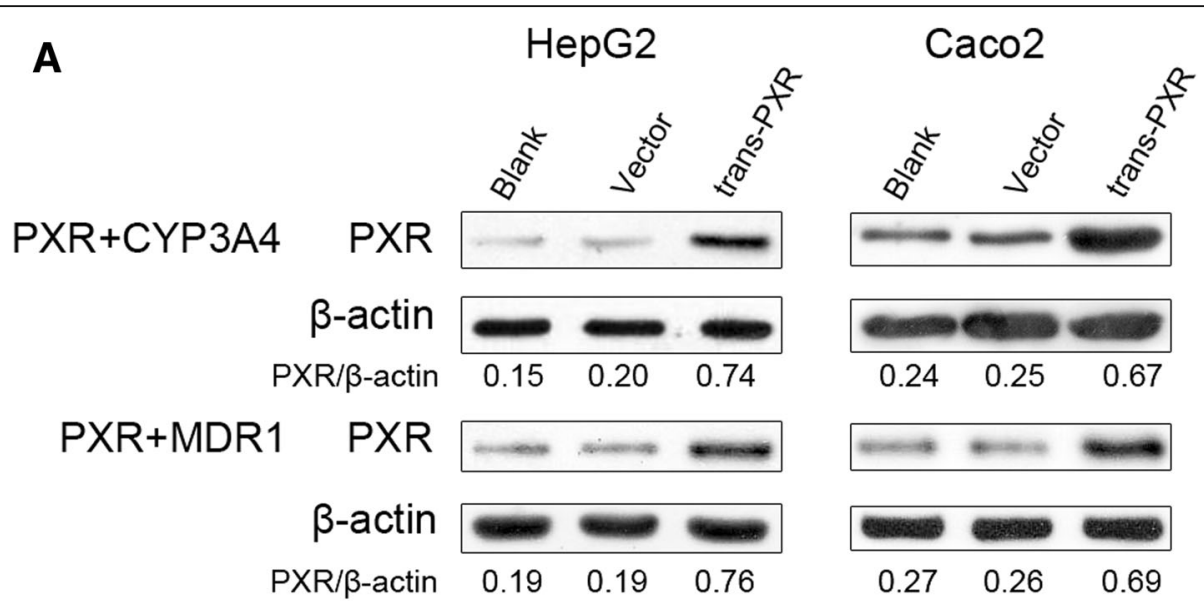

B

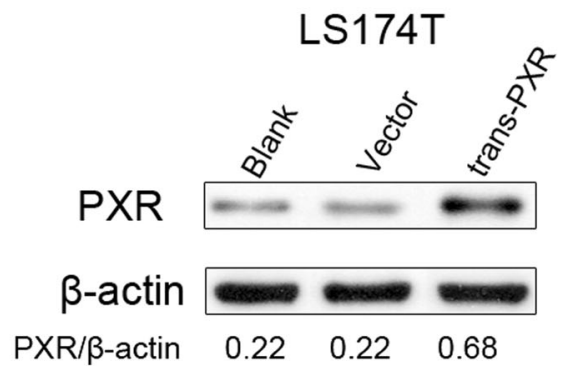

Fig. 2 The expression of PXR fusion protein in vitro was detected by Western blotting. a Blank: cells were non-transfected, Vector: cells were transfected with 600 ng CYP3A4 (or MDR1) + 100 ng pcDNA3.1 + 10 ng PRL-SV40, trans-PXR: cells transfected with 600 ng CYP3A4 (or MDR1) + 100 ng pcDNA3.1-PXR + 10 ng PRL-SV40. b Blank: cells were non-transfected, Vector: cells were transfected with 100 ng pcDNA3.1 + 10 ng PRL-SV40, trans-PXR: cells were transfected with $100 \mathrm{ng}$ PCDNA3.1-PXR + $10 \mathrm{ng}$ PRL-SV40

hPXR in HepG2 and Caco2 cells. We successfully transfected PXR-MDR1 plasmid constructs in HepG2 and Caco2 cells (Fig. 2). Twenty-four hours following transfection, HepG2 and Caco2 cells were separately treated with rifampicin $(10 \mu \mathrm{M}, \mathrm{hPXR}$ agonist), PCN $(10 \mu \mathrm{M}$, rPXR agonist), SGD $(0.1,1$, and $2 \mathrm{mg} / \mathrm{ml}), \mathrm{SY}(0.05,0.5$, and $1 \mathrm{mg} / \mathrm{ml})$ and GC $(0.05,0.5$, and $1 \mathrm{mg} / \mathrm{ml})$ for $24 \mathrm{~h}$, detected dual luciferase activity.

Results are shown in Fig. 4. In hPXR-transfected HepG2 and Caco2 cells, rifampicin, SGD and its constituent prescription GC significantly increase the luciferase activities of MDR1 when compared with $0.1 \%$ DMSO group $(p<0.05)$. However, SY only shows the significant increase in luciferase activities of transfected HepG2 cells. The increases of the three decoctions in MDR1 luciferase activities also show a concentration-dependent trend. In PXR-HepG2 cells, MDR1 luciferase activity is significantly increased by rifampicin (22.90), $0.1 \mathrm{mg} / \mathrm{ml}$ SGD (8.10), $2 \mathrm{mg} / \mathrm{ml} \mathrm{SGD}$ (12.80), SY (4.60, 5.25, 6.04), GC (13.83, 15.14, 17.10) when compared with DMSO, respectively. In PXR-Caco2 cells, the luciferase activity of MDR1 is significantly increased by rifampicin (24.10), $1 \mathrm{mg} / \mathrm{ml} \mathrm{SGD}$ (11.71), $2 \mathrm{mg} / \mathrm{ml} \mathrm{SGD} \mathrm{(12.19)} \mathrm{and} \mathrm{GC} \mathrm{(14.86,} \mathrm{15.43,}$
17.33) when compared with DMSO. However, in HepG2-hPXR and Caco2-hPXR cells, PCN treatment doesn't cause a significant increase in MDR1 luciferase activities when compared with the DMSO group.

In a word, in HepG2 and Caco2 cells, SGD and its constituent prescription GC significantly increase promoter activities of CYP3A4 and MDR1 via PXR in a concentration-dependent manner. While SY doesn't significantly increase promoter activities of MDR1 in transfected Caco2 cells. Like rifampicin, $1 \mathrm{mg} / \mathrm{ml} \mathrm{GC}$ is a strong agonist of PXR, followed by SGD, and SY ranges the weakest.

\section{SGD and its constituent herbs upregulate expression of CYP3A4 and MDR1 mRNA in LS174T via PXR}

Finally, to verify effects of SGD and its constituent herbs on CYP3A4 and MDR1 mRNA expression levels by PXR activation, we used PXR-transfected LS174T cell lines for real-time qPCR (Fig. 5). LS174T cells express endogenous hPXR with inducible activity [32], which was supported by our observation of a low PXR protein expression in LS174T (Fig. 2B). Twenty-four hours following transfection, LS174T cells were exposed to phenobarbital (1 mM, PXR agonist), $1 \mathrm{mg} / \mathrm{ml}$ SGD, SY 

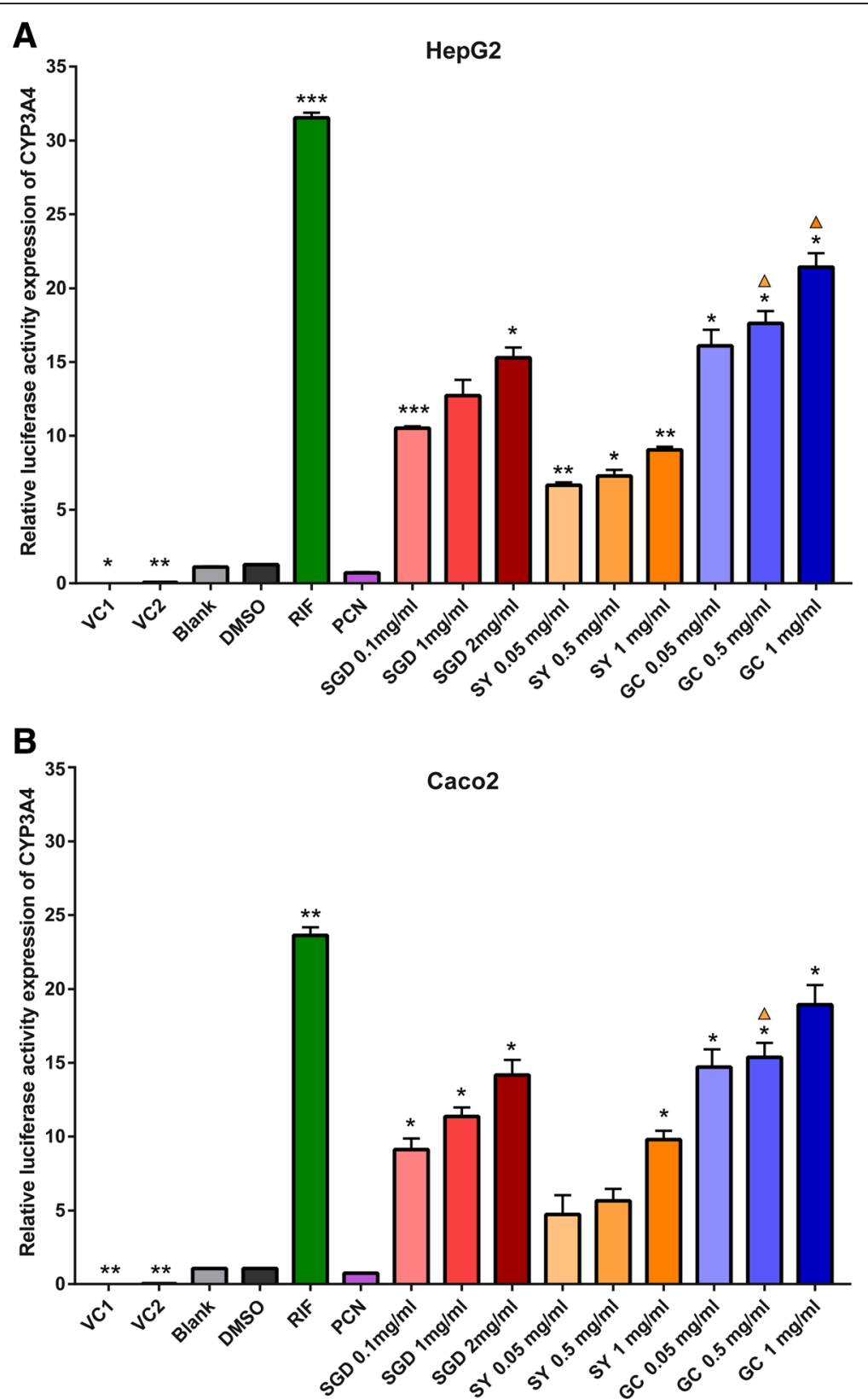

Fig. 3 Induced effects of CYP3A4 promoter mediated via PXR by SGD, SY and GC in HepG2 (a) and Caco2 (b). There is a significant increase in positive control RIF group and most prescriptions groups when compared with 0.1\% DMSO treatment group $(p<0.05)$. There is no significant decrease in negative control PCN group when compared to the DMSO group $(p>0.05)$. VC1: cells were transfected with pcDNA3.1-PXR, PRL-SV40, pGL4.17; VC2: cells were transfected with pCDNA3.1, PRL-SV40, pGL4.17-CYP3A4; other groups: cells were transiently transfected with pCDNA3.1-PXR, PRL-SV40, pGL4.17CYP3A4. All data were obtained from three independent experiments performed by triplicate and expressed as mean \pm S.E.M. Statistical significance was determined by one-way ANOVA with Welch correction, followed by the Games Howell test for pairwise comparisons. One-way ANOVA with Welch's correction revealed significant differences among the groups (Welch's $p<0.001$ ). ${ }^{*} p<0.05,{ }^{* *} p<0.01,{ }^{* * *} p<0.001$ compared with the DMSO group; $\mathbf{\Delta} p<$ 0.05 represents GC group compared to SY group with the same concentration

and GC for $48 \mathrm{~h}$, separately. In order to avoid the effect of herbal concentration, the same concentration was adopted. In LS174T cells with non-transfected or blank vector-transfected, phenobarbital, SGD, SY and GC significantly up-regulate level of CYP3A4 and MDR1 mRNA compared with corresponding DMSO groups $(p<0.05)$.
And GC has a significant up-regulation when compared to SY $(p<0.01)$. Significantly enhancements of CYP3A4 and MDR1 mRNA expression are also observed in PXR-transfected cells. This indicates phenobarbital, SGD, SY and GC may activate endogenous and exogenous PXR and then significantly up-regulate 


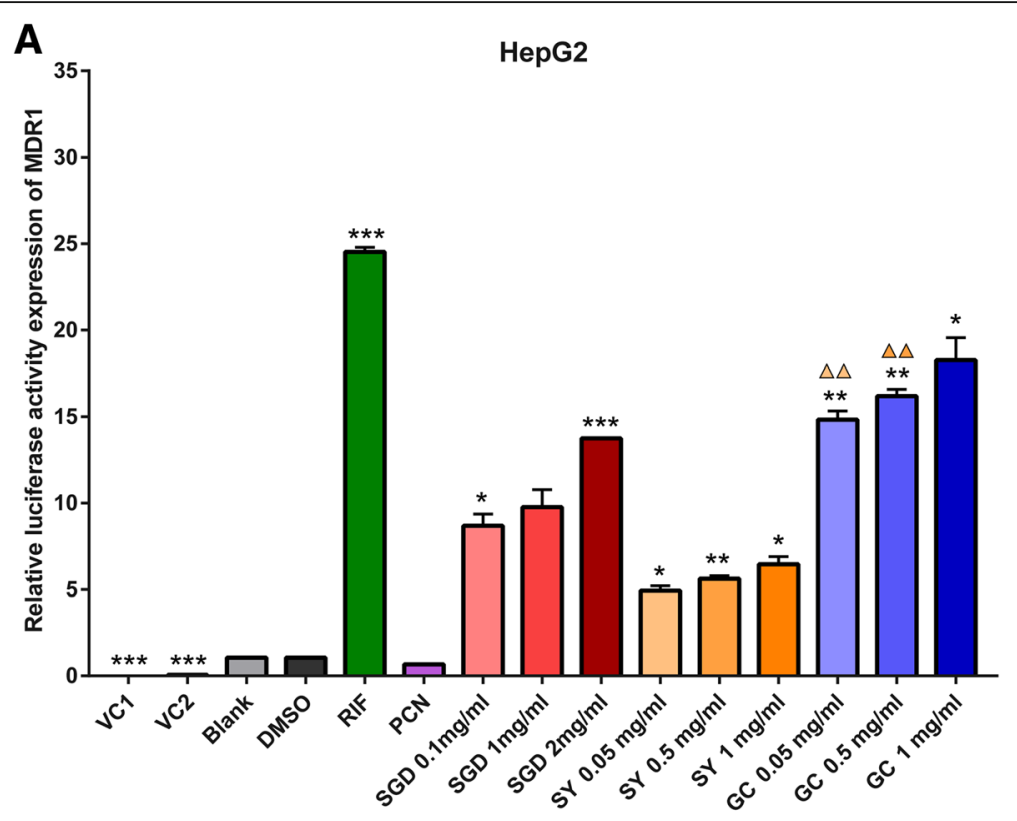

B

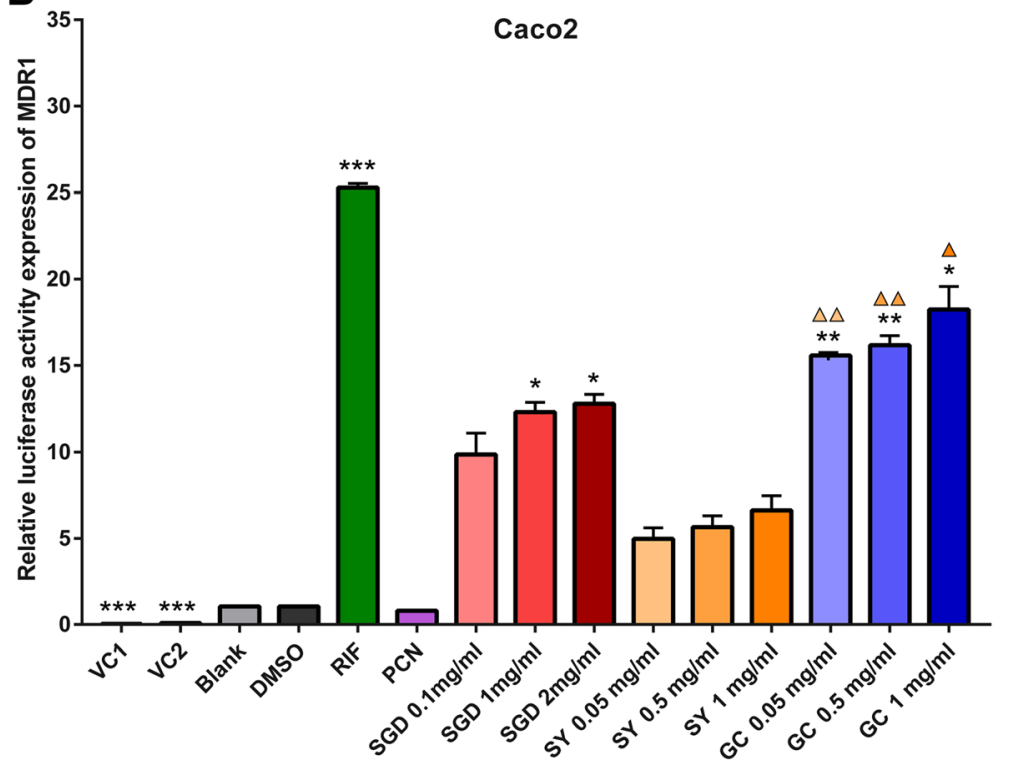

Fig. 4 Induced effects of MDR1 promoter mediated via PXR by SGD, SY and GC in HepG2 (a) and Caco2 (b). There is a significant increase in positive control RIF group and most prescriptions groups when compared with $0.1 \%$ DMSO treatment group $(p<0.05)$. There is no significant decrease in negative control PCN group when compared to the DMSO group $(p>0.05)$. VC1: cells were transfected with pCDNA3.1-PXR, PRLSV40, pGL4.17; VC2: cells were transfected with PCDNA3.1, PRL-SV40, PGL4.17-CYP3A4; other groups: cells were transiently transfected with pCDNA3.1-PXR, PRL-SV40, pGL4.17-CYP3A4. All data were obtained from three independent experiments performed by triplicate and expressed as mean \pm S.E.M. Statistical significance was determined by one-way ANOVA with Welch correction, followed by the Games Howell test. One-way ANOVA with Welch's correction revealed significant differences among the groups (Welch's $p<0.001$ ). ${ }^{*} p<0.05,{ }^{* *} p<0.01$, ${ }^{* * *} p<0.001$ compared with the DMSO group; $\boldsymbol{\Delta} p<0.05, \boldsymbol{\Delta} \mathbf{\Delta} p<0.01$ represents GC group compared to SY group with the same concentration

CYP3A4 and MDR1 mRNA expression in LS174T cells. In PXR-transfected groups, increases of CYP3A4 and MDR1 mRNA expression are significantly higher by phenobarbital, SGD, SY, GC than them in non-transfected groups or vector-transfected groups $(p<0.01)$. All of that means SGD and its constituent prescriptions up-regulate expression of CYP3A4 and MDR1 mRNA in LS174T via PXR.

\section{Discussion}

The present study demonstrates that SGD, SY and GC result in a concentration-dependent increase in CYP3A4 

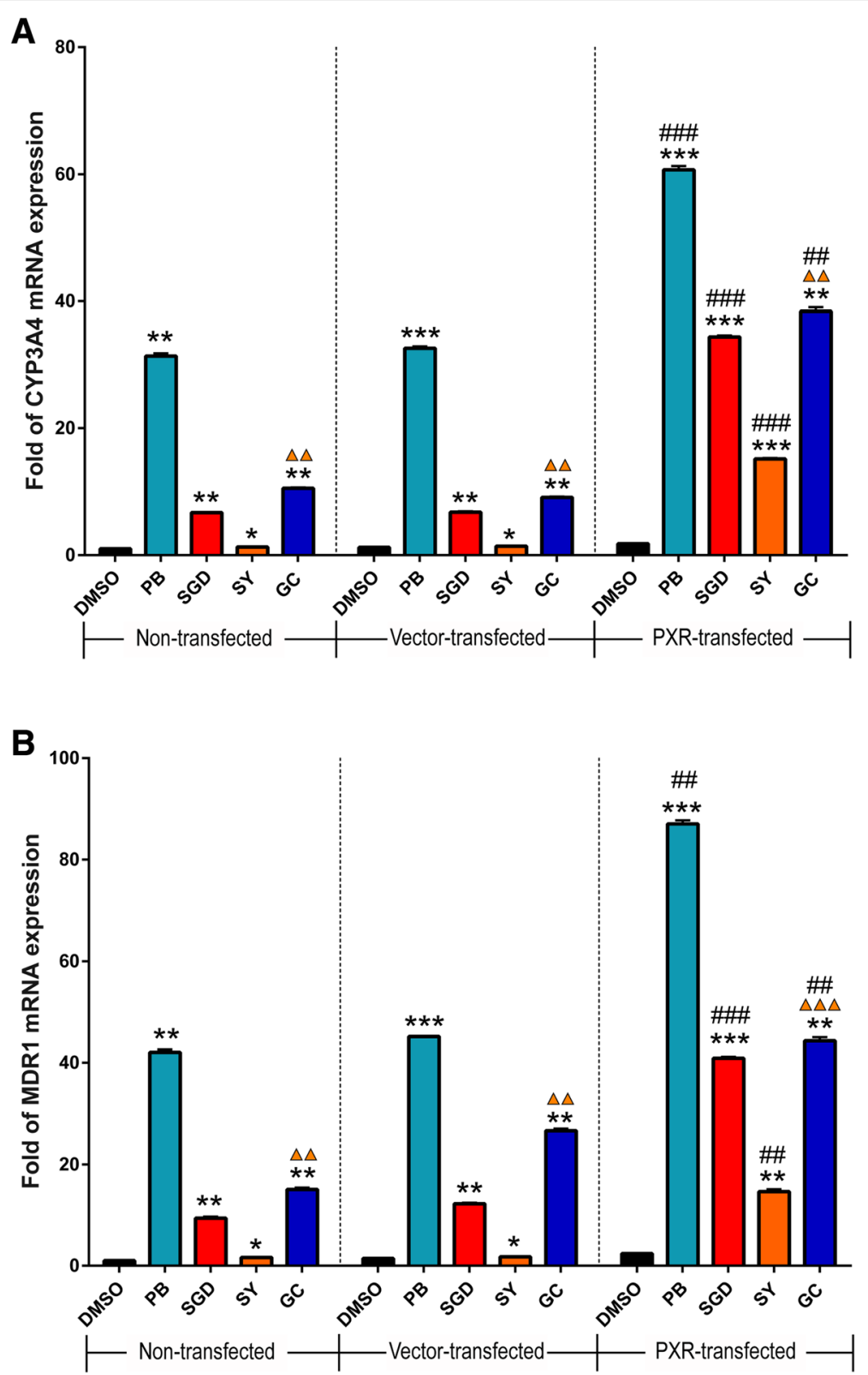

Fig. 5 The up-regulation of phenobarbital (PB), SGD, SY, GC on CYP3A4 (a) and MDR1 (b) mRNA expression. LS174T cells were transfected with $400 \mathrm{ng} /$ well pcDNA3.1-PXR, or pcDNA3.1 vector, or weren't transfected. Cells were then exposed to phenobarbital (1 mM), or SGD (1 mg/ml), or SY $(1 \mathrm{mg} / \mathrm{ml})$, or GC $(1 \mathrm{mg} / \mathrm{ml})$ for $48 \mathrm{~h}$. mRNA expression of CYP3A4 and MDR1 was determined using real-time qPCR. Data are presented as -fold increase to non-transfected group with DMSO treatment. Data are presented as means \pm S.E.M of three independent experiments performed in triplicates. Statistical significance was determined by one-way ANOVA with Welch correction, followed by the Games Howell test. One-way ANOVA with Welch's correction revealed significant differences among the groups (Welch's $p<0.001$ ). ${ }^{*} p<0.05,{ }^{* *} p<0.01$, ${ }^{* * *} p<0.001$ compared to the DMSO group with the same transfection; $\boldsymbol{\Delta} \mathbf{\Delta} p<0.01, \mathbf{\Delta} \boldsymbol{\Delta} \mathbf{\Delta} p<0.001$ represents GC group compared to SY group with the same transfection; \#\#p $<0.01$, \#\#\# $<0.001$ compared to vector-transfected group with the same drug treatment

and MDR1 promoter activity in HepG2 and Caco2 cells via PXR activation after $24 \mathrm{~h}$ exposure. Moreover, we show that SGD, SY and GC are capable of up-regulating CYP3A4 and MDR1 gene expression in LS174T through activation of PXR pathway. Interestingly, Gancao makes the main contribution to SGD on the induction. The illustration is shown in Fig. 6.
Chinese herbal medicine has been shown to have synergistic actions with chemotherapy and reduce their side effects [5]. An overall prevalence of combined utilization is estimated up to $63 \%$ among cancer patients in the United States [3]. However, with the increasing co-administration of herbal medicine with chemotherapy, herb-drug interactions have become an important 


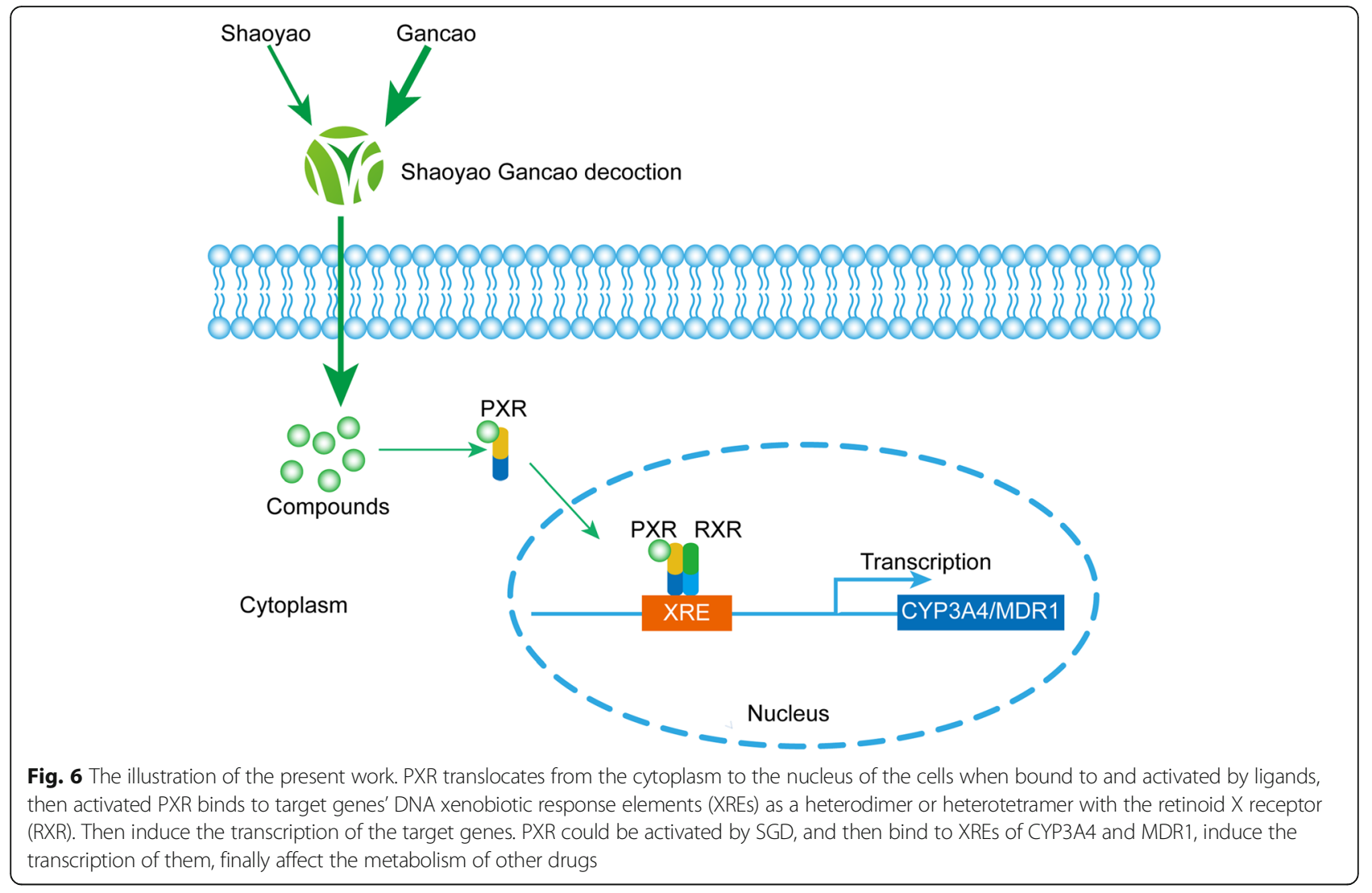

issue in clinical practice. It has been found that traditional herbal formulas cause clinically-relevant interactions and affect the metabolism and pharmacokinetics of chemotherapeutic agents $[3,36,37]$. In the previous study, we observed that pretreatment with SGD for 14 days significantly altered pharmacokinetics of paclitaxel involved in decreasing the area under the curve and increasing the total clearance of intravenous paclitaxel in rats [15]. In general, the reduction in area under the curve and increase of the clearance after co-administration is due to the induction of the metabolic enzyme of drugs and/or the drug transporters [20]. However, studies of SGD on drug metabolic enzymes and drug transporters are rare so far. Therefore, it is urgent to evaluate effects of SGD on drug metabolic enzymes and drug transporters and to uncover the underlying molecular mechanism.

The CYP450 enzyme system is responsible for the biotransformation of more than $90 \%$ of the drugs on the market [38]. The inducers of CYP450 can speed up the metabolism of substrates and drugs, including most chemotherapeutic drugs [30]. And the largest fraction of the P450 reactions is catalyzed by CYP3A4 [18], which is mainly distributed in the liver and intestine and metabolizes at least $50 \%$ of marketed pharmaceutical agents [39]. MDR1 encodes P-glycoprotein, an important transmembrane efflux pump protein, to limit the absorption of drugs and contribute to the excretion of drugs in the intestine [32]. The induction of many CYPs and drug transporters occurs by a similar mechanism, where ligand activation of PXR [34]. PXR could be activated by ligands and translocate from the cytoplasm to the nucleus [34]. Then activated PXR binds to specific promoter responsive elements of many xenobiotic enzymes and transporter genes as a heterodimer or heterotetramer with the retinoid $X$ receptor (RXR), thus, induces the transcription [40-42]. Here, we established SGD and its constituent herbs (SY and GC) as natural potent agonists of PXR and proved that they induce the transcription of intestinal and hepatic CYP3A4 and MDR1 via PXR activation in vitro. Those in vitro results might partially account for the pharmacokinetics alteration of drugs via SGD in vivo.

SGD, an antispasmodic herbal formula, is consisted of Shaoyao (Paeoniae Radix Alba) and Gancao (Glycyrrhizae Radix et Rhizoma). It is widely used in Asia to treat muscle cramps, many kinds of pain, abdominal spasmodic pain, and dysmenorrhea [10]. The previous study has shown that the combination of Shaoyao and Gancao might work "synergistically" to improve the efficacy or reduce the toxicity of individual components [43]. The contribution of constituent herbs should also be 
assessed. In the present study, the induction of CYP3A4 and MDR1 by SY and GC is in accordance with previous reports $[25,26,44,45]$. The present study also showed the induction of CYP3A4 and MDR1 via Gancao was much higher than Shaoyao. That indicates Gancao is the main contributor to SGD on the induction of CYP3A4 and MDR1. Excess of SGD or Gancao can also lead to a failure of most drugs since they enhance the drug metabolizing enzymes which could lead to a rapid removal of the drugs before its therapeutic action. SGD or Gancao should be administrated with caution.

In most cases, it is not good for PXR to be activated. Conversely, the activation of PXR by herbs may have favorable effects. Recent studies have revealed much more complex regulatory pathways governed by PXR, such as lipid and glucose metabolism, bile acid metabolism and inflammation [34]. PXR activation prevents lithocholic acid-induced hepatotoxicity, suggesting that PXR agonists may be useful in the treatment of human cholestatic liver disease [46]. A strong inducer of PXR St. John's wort significantly affects serum concentrations of irinotecan, a chemotherapeutic agent, consequently reducing the antineoplastic effect and toxicity of this CYP3A4 substrate [47]. Overall, these studies highlight the dual features of PXR activation: promoting drug metabolism leads to serious potential drug interactions and treatment failures, and activation of the detoxification system helps protect our body from invading of toxic substances. Therefore, when prescribing herbs with agents, it is necessary to monitor the plasma concentration of agents to guarantees effectiveness and reduce toxicity.

The limitations of the present study are clear, and further explorations in the future are demanded. Firstly, our study only focuses on the activation of PXR. Beyond PXR, more nuclear receptors, such as CAR, are needed to be studied in the future. Besides, we illustrate the up-regulation of SGD on CYP3A4 and MDR1 via PXR activation, we didn't evaluate effects of active compounds from SGD.

\section{Conclusions}

This study firstly observed SGD induce intestinal and hepatic CYP3A4 and MDR1 promoter and enhance mRNA expression via activating PXR pathway in vitro. And Gancao plays a predominant role in the induction effects. This study highlights a potential of SGD on the PXR-mediated regulation of drug metabolic enzymes and drug transporters.

\section{Abbreviations}

CYP450: Cytochrome P450; DEPC: Diethyl pyrocarbonate; DMEM: Dulbecco's modified eagle medium; DMSO: Dimethyl sulfoxide; FBS: fetal bovine serum; GC: Gancao; MDR1: Multidrug resistance protein 1; PB: Phenobarbital;

PCN: Pregnenolone 16a-carbonitrile; PXR: Pregnane $X$ receptor;
RIF: Rifampicin; SGD: Shaoyao Gancao decoction; SY: Shaoyao; UPLC: Ultraperformance liquid chromatography; VC: Vector control

\section{Acknowledgements}

We would like to thank Professor Guo Wang (Institute of Clinical Pharmacology, Central South University, Changsha, China) and his colleagues for their kindly gift of the human PXR expression plasmid constructs.

\section{Funding}

This work was supported by the Natural Science Foundation of China (Grant Nos. 81202781, 81403259, 81774322, 81673719 and 81473573), China Postdoctoral Science Foundation (No. 2017T100614) and the Fundamental Research Funds for the Central Universities (2012QNZT110).

Availability of data and materials

All data generated or analyzed in this study are included in this manuscript.

\section{Authors' contributions}

$D F, H C, Y W, P G$ designed the study, conducted experiments, analyzed the data and prepared the manuscript. $T, R F, J L$ reviewed and corrected the manuscript. All authors read and approved the final manuscript.

Ethics approval and consent to participate

Not applicable.

Consent for publication

Not applicable.

\section{Competing interests}

The authors declare that they have no competing interests.

\section{Publisher's Note}

Springer Nature remains neutral with regard to jurisdictional claims in published maps and institutional affiliations.

Received: 3 May 2018 Accepted: 6 December 2018

Published online: 29 December 2018

\section{References}

1. Mok TS, Yeo W, Johnson PJ, Hui P, Ho WM, Lam KC, Xu M, Chak K, Chan A, Wong $\mathrm{H}$, et al. A double-blind placebo-controlled randomized study of Chinese herbal medicine as complementary therapy for reduction of chemotherapy-induced toxicity. Ann Oncol. 2007:18(4):768-74.

2. Lin TH, Yen HR, Chiang JH, Sun MF, Chang HH, Huang ST. The use of Chinese herbal medicine as an adjuvant therapy to reduce incidence of chronic hepatitis in colon cancer patients: a Taiwanese population-based cohort study. J Ethnopharmacol. 2017:202:225-33.

3. Sparreboom A, Cox MC, Acharya MR, Figg WD. Herbal remedies in the United States: potential adverse interactions with anticancer agents. J Clin Oncol. 2004;22(12):2489-503.

4. Ben-Arye E, Lavie O, Samuels N, Khamaisie H, Schiff E, Raz OG, Mahajna J. Safety of herbal medicine use during chemotherapy in patients with ovarian cancer: a "bedside-to-bench" approach. Med Oncol. 2017;34(4):54.

5. Lam W, Bussom S, Guan F, Jiang Z, Zhang W, Gullen EA, Liu SH, Cheng YC. The four-herb Chinese medicine PHY906 reduces chemotherapy-induced gastrointestinal toxicity. Sci Transl Med. 2010;2(45):45ra59.

6. Hung KF, Hsu CP, Chiang JH, Lin HJ, Kuo YT, Sun MF, Yen HR Complementary Chinese herbal medicine therapy improves survival of patients with gastric cancer in Taiwan: a nationwide retrospective matchedcohort study. J Ethnopharmacol. 2017;199:168-74.

7. Farrell MP, Kummar S. Phase I/IIA randomized study of PHY906, a novel herbal agent, as a modulator of chemotherapy in patients with advanced colorectal cancer. Clin Colorectal Cancer. 2003:2(4):253-6.

8. Gunji S, Ueda S, Yoshida M, Kanai M, Terajima H, Takabayashi A. Effects of rikkunshito, a kampo medicine, on quality of life after proximal gastrectomy. J Surg Res. 2013;185(2):575-80.

9. Yoshida T, Sawa T, Ishiguro T, Horiba A, Minatoguchi S, Fujiwara H. The efficacy of prophylactic Shakuyaku-Kanzo-to for myalgia and arthralgia following carboplatin and paclitaxel combination chemotherapy for nonsmall cell lung cancer. Support Care Cancer. 2009;17(3):315-20. 
10. Wang $Y$, Xu C, Wang $P$, Lin $X$, Yang Y, Li D, Li H, Wu X, Liu H. Pharmacokinetic comparisons of different combinations of ShaoyaoGancao-decoction in rats: simultaneous determination of ten active constituents by HPLC-MS/MS. J Chromatogr B Analyt Technol Biomed Life Sci. 2013;932:76-87.

11. Hinoshita F, Ogura Y, Suzuki Y, Hara S, Yamada A, Tanaka N, Yamashita A, Marumo F. Effect of orally administered shao-Yao-Gan-cao-tang (Shakuyakukanzo-to) on muscle cramps in maintenance hemodialysis patients: a preliminary study. Am J Chin Med. 2003;31(3):445-53.

12. Yamamoto K, Hoshiai H, Noda K. Effects of shakuyaku-kanzo-to on muscle pain from combination chemotherapy with paclitaxel and carboplatin. Gynecol Oncol. 2001;81(2):333-4.

13. Hidaka T, Shima T, Nagira K, leki M, Nakamura T, Aono Y, Kuraishi Y, Arai T, Saito S. Herbal medicine Shakuyaku-kanzo-to reduces paclitaxel-induced painful peripheral neuropathy in mice. Eur J Pain. 2009;13(1):22-7.

14. Fujii K, Okamoto S, Saitoh K, Sasaki N, Takano M, Tanaka S, Kudoh K, Kita T, Tode T, Kikuchi Y. The efficacy of Shakuyaku-Kanzo-to for peripheral nerve dysfunction in paclitaxel combination chemotherapy for epithelial ovarian carcinoma. Gan To Kagaku Ryoho. 2004;31(10):1537-40.

15. Wang Y, Huang X, Zhong MZ, Lu RH, Xia ZA, Fan R, Liu B, Huang W, Gan PP. Pretreatment of Shaoyao Gancao decoction alters pharmacokinetics of intravenous paclitaxel in rats. Chin J Integr Med. 2017;23(1):70-5.

16. Qin CZ, Lv QL, Wu NY, Cheng L, Chu YC, Chu TY, Hu L, Cheng Y, Zhang X, Zhou HH. Mechanism-based inhibition of Alantolactone on human cytochrome P450 3A4 in vitro and activity of hepatic cytochrome P450 in mice. J Ethnopharmacol. 2015;168:146-9.

17. Lv QL, Wang GH, Chen SH, Hu L, Zhang X, Ying G, Qin CZ, Zhou HH. In vitro and in vivo inhibitory effects of Glycyrrhetinic acid in mice and human cytochrome P450 3A4. Int J Environ Res Public Health. 2015;13(1):84

18. Rendic S, Guengerich FP. Survey of human oxidoreductases and cytochrome P450 enzymes involved in the metabolism of xenobiotic and natural chemicals. Chem Res Toxicol. 2015;28(1):38-42.

19. Lim YP, Ma CY, Liu CL, Lin YH, Hu ML, Chen JJ, Hung DZ, Hsieh WT, Huang JD. Sesamin: a naturally occurring Lignan inhibits CYP3A4 by antagonizing the Pregnane $X$ receptor activation. Evid Based Complement Alternat Med 2012; 2012:242810.

20. Sahi J, Milad MA, Zheng X, Rose KA, Wang H, Stilgenbauer L, Gilbert D, Jolley S, Stern RH, LeCluyse EL. Avasimibe induces CYP3A4 and multiple drug resistance protein 1 gene expression through activation of the pregnane X receptor. J Pharmacol Exp Ther. 2003;306(3):1027-34.

21. Geick A, Eichelbaum M, Burk O. Nuclear receptor response elements mediate induction of intestinal MDR1 by rifampin. J Biol Chem. 2001; 276(18):14581-7.

22. Burk O, Arnold KA, Nussler AK, Schaeffeler E, Efimova E, Avery BA, Avery MA, Fromm MF, Eichelbaum M. Antimalarial artemisinin drugs induce cytochrome P450 and MDR1 expression by activation of xenosensors pregnane $X$ receptor and constitutive androstane receptor. Mol Pharmacol. 2005;67(6):1954-65.

23. Mu Y, Zhang J, Zhang S, Zhou HH, Toma D, Ren S, Huang L, Yaramus M Baum A, Venkataramanan R, et al. Traditional Chinese medicines Wu Wei Zi (Schisandra chinensis Baill) and Gan Cao (Glycyrrhiza uralensis Fisch) activate pregnane $X$ receptor and increase warfarin clearance in rats. J Pharmacol Exp Ther. 2006;316(3):1369-77.

24. Yu C, Chai X, Yu L, Chen S, Zeng S. Identification of novel pregnane $X$ receptor activators from traditional Chinese medicines. J Ethnopharmacol. 2011;136(1):137-43.

25. Chen Y, Wang J, Wang L, Chen L, Wu Q. Absorption and interaction of the main constituents from the traditional Chinese drug pair Shaoyao-Gancao via a Caco-2 cell monolayer model. Molecules. 2012;17(12):14908-17.

26. Liu LL, Guan YM, Lu XP, Liang XL, Chen LH. Mechanisms of P-glycoprotein modulation by semen Strychni combined with Radix Paeoniae Alba. Evid Based Complement Alternat Med. 2017;2017:1743870.

27. Gan PP, Zhong MZ, Huang $X$, Sun M, Wang Y. Determination of six major compounds in Shaoyao-Gancao-Tang and its single herb decoctions. Asian J Chem. 2011;23(4):1515-9.

28. Gan PP, Huang X, Zhong MZ, Sun M, Qin F, Zhang CH. Simultaneous determination of eight major constituents in the traditional Chinese medicine Shaoyao-Gancao - Tang by UPLC-PDA. J Med Plants Res. 2010; 4(24):2615-21.

29. Gan $P$, Zhong M, Huang $X$, Sun M, Wang Y, Xiao Y, Zeng C, Yuan Q, Liu Z, Zhou H. Pharmacokinetic comparisons of albiflorin and paeoniflorin after oral administration of Shaoyao-Gancao-Tang and single herb Paeony decoction to rats. Planta Med. 2012;78(3):237-43.

30. Chen Q, Xie HT, Li Y, Wang G, Xu Z, Pu ZC, Hu H. Transcriptional regulation of CYP3A4/2B6/2C9 mediated via nuclear receptor PXR by Helicid and its metabolites. Evid Based Complement Alternat Med. 2015;2015:797496.

31. Livak KJ, Schmittgen TD. Analysis of relative gene expression data using real-time quantitative PCR and the 2(-Delta Delta $C(T))$ method. Methods. 2001;25(4):402-8.

32. Wang YM, Lin W, Chai SC, Wu J, Ong SS, Schuetz EG, Chen T. Piperine activates human pregnane $X$ receptor to induce the expression of cytochrome P450 3A4 and multidrug resistance protein 1. Toxicol Appl Pharmacol. 2013;272(1):96-107.

33. Jones SA, Moore LB, Shenk JL, Wisely GB, Hamilton GA, McKee DD, Tomkinson NC, LeCluyse EL, Lambert MH, Willson TM, et al. The pregnane X receptor: a promiscuous xenobiotic receptor that has diverged during evolution. Mol Endocrinol. 2000;14(1):27-39.

34. Chai X, Zeng S, Xie W. Nuclear receptors PXR and CAR: implications for drug metabolism regulation, pharmacogenomics and beyond. Expert Opin Drug Metab Toxicol. 2013;9(3):253-66.

35. Martin P, Riley R, Back DJ, Owen A. Comparison of the induction profile for drug disposition proteins by typical nuclear receptor activators in human hepatic and intestinal cells. Br J Pharmacol. 2008;153(4):805-19.

36. Yap KY, See CS, Chan A. Clinically-relevant chemotherapy interactions with complementary and alternative medicines in patients with cancer. Recent Pat Food Nutr Agric. 2010;2(1):12-55.

37. Zhang $X$, Zheng $W$, Xu H, Huang $X$, Ren $P$, Zou H, Liu G, Wang J, Ma X. Pharmacokinetic study of representative anti-oxidative compounds from Denshen-chuanxiong-Honghua following oral administration in rats. J Chromatogr B Analyt Technol Biomed Life Sci. 2017;1052:82-90.

38. de Groot MJ. Designing better drugs: predicting cytochrome P450 metabolism. Drug Discov Today. 2006;11(13-14):601-6.

39. Vachirayonstien T, Yan B. MicroRNA-30c-1-3p is a silencer of the pregnane $X$ receptor by targeting the $3^{\prime}$-untranslated region and alters the expression of its target gene cytochrome P450 3A4. Biochim Biophys Acta. 2016; 1859(9):1238-44.

40. Wang $\mathrm{H}$, LeCluyse EL. Role of orphan nuclear receptors in the regulation of drug-metabolising enzymes. Clin Pharmacokinet. 2003:42(15):1331-57.

41. Nannelli A, Rossignolo F, Tolando R, Rossato P, Pellegatti M, Longo V, Gervasi PG. Expression and distribution of CYP3A genes, CYP2B22, and MDR1, MRP1, MRP2, LRP efflux transporters in brain of control and rifampicin-treated pigs. Mol Cell Biochem. 2010;337(1-2):133-43.

42. Cerveny L, Svecova L, Anzenbacherova E, Vrzal R, Staud F, Dvorak Z, Ulrichova J, Anzenbacher P, Pavek P. Valproic acid induces CYP3A4 and MDR1 gene expression by activation of constitutive androstane receptor and pregnane $X$ receptor pathways. Drug Metab Dispos. 2007;35(7):1032-41.

43. Shen L, Hu RW, Lin X, Cong WJ, Hong YL, Feng Y, Xu DS, Ruan KF. Pharmacokinetics of characteristic effective ingredients from individual and combination Shaoyao and Gancao treatment in rats using HPLC fingerprinting. Eur J Drug Metab Pharmacokinet. 2012;37(2):133-40.

44. Hou YC, Lin SP, Chao PD. Liquorice reduced cyclosporine bioavailability by activating P-glycoprotein and CYP 3A. Food Chem. 2012;135(4):2307-12.

45. Tang J, Song $X$, Zhu M, Zhang J. Study on the pharmacokinetics drug-drug interaction potential of Glycyrrhiza uralensis, a traditional Chinese medicine, with lidocaine in rats. Phytother Res. 2009;23(5):603-7.

46. Staudinger JL, Goodwin B, Jones SA, Hawkins-Brown D, MacKenzie Kl, LaTour A, Liu Y, Klaassen CD, Brown KK, Reinhard J, et al. The nuclear receptor PXR is a lithocholic acid sensor that protects against liver toxicity. Proc Natl Acad Sci U S A. 2001;98(6):3369-74.

47. Hu ZP, Yang XX, Chen $X$, Cao J, Chan E, Duan W, Huang $M$, Yu XQ, Wen JY, Zhou SF. A mechanistic study on altered pharmacokinetics of irinotecan by St. John's wort. Curr Drug Metab. 2007:8(2):157-71. 